\title{
Study on Joint Inventory Management in Supply Chain
}

\author{
Yujing Wang ${ }^{1}$, Keming Zhang ${ }^{2}$ \\ ${ }^{1}$ School of Wang Yujing, Beijng Jiaotong University, Beijing, China; \\ ${ }^{2}$ School of Zhang KeMing, Beijng Jiaotong University, Beijing, China.
}

Keywords: Joint, Inventory Management, Supply Chain.

\begin{abstract}
Nowadays, the competition between enterprises has gradually transformed into the competition among supply chains, and the cost of inventory occupies a large proportion in the whole supply chain management. The traditional inventory control method is that enterprises should act as their own means and use their own strategies to manage the inventory and have a single inventory management method. This results in problems such as high overall inventory level of the supply chain.
\end{abstract}

\section{Introduction}

Because supply chain information is not shared in all aspects, it is easy to cause distortion of demand information, resulting in the bullwhip effect. To solve these problems, methods such as VMI and JMI have emerged.

VMI is a more advanced inventory management method, which reflects the integrated management of supply chain, but VMI also has its own limitations:

(1) The overall level of VMI collaboration is limited;

(2) VMI does not achieve sufficient information sharing.

(3) The implementation of VMI can reduce the total cost of inventory, but in the VMI system, transportation costs and inventory costs are all borne by supplier, which undoubtedly increases the risk of supplier.

Therefore, in order to overcome the limitations of VMI and the bullwhip effect of traditional inventory control methods, a joint inventory management (JIM) approach has emerged.

\section{The Concept of JMI and Its Implementation Strategy}

\subsection{The Concept of JMI.}

VMI is a kind of decision-making proxy mode of supply chain integrated operation. Unlike VMI, joint inventory management is a kind of inventory management mode that shares the responsibility and risk between supplier and user. JMI stressed that starting from the overall supply chain, the supply chain upstream and downstream to jointly negotiate and jointly develop inventory control plan, and then plans to be released to the manufacturing unit and the implementation of the sales unit, thus to solve the inventory problem in the various nodes. The needs are determined by the both side in the supply chain considering the coordination between supply and demand, making inventory management is no longer an independent operation of their own way. While, JMI becomes the connection link between the supply and demand sides, it also can improve the overall operational efficiency.

JMI reflects the high degree of collaboration among the nodes in the supply chain. The supply chain organizations not only share demand information in real time, but also establish a complete set of coordination management mechanisms, which can not only balance the cost and benefit risks of the whole supply chain, but also make it possible to clearly allocate the costs, risks and benefits of all parties in the supply chain. In addition to stimulating the various organizations, JMI also avoids the acts that all parties have done to undermine the interests of other members, thereby greatly enhancing the stability of the supply chain. 


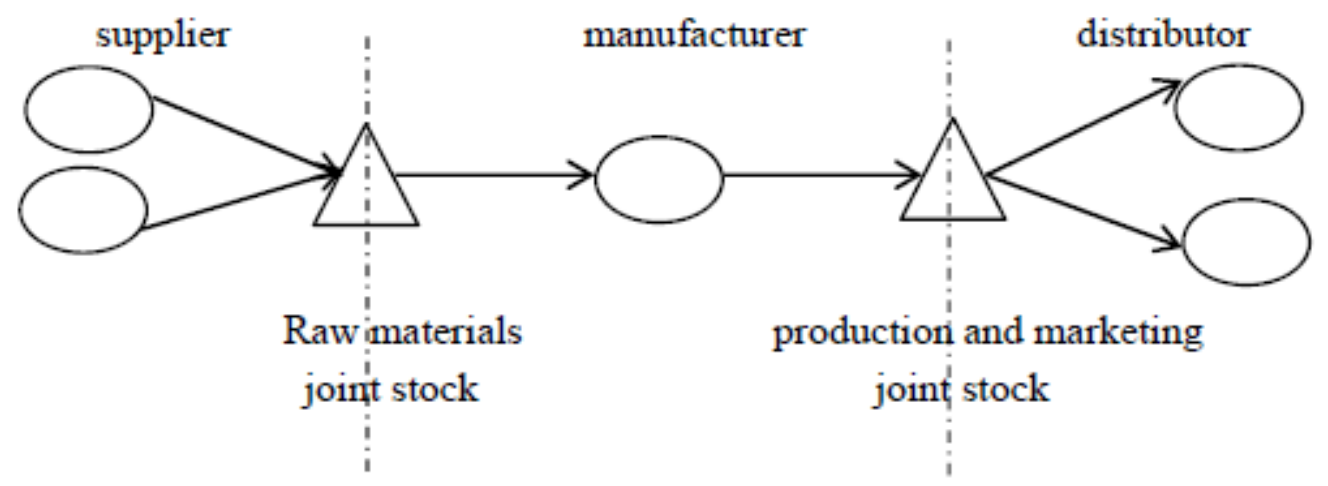

Figure 1. JMI model base on supply chain

Traditional inventory control method is each enterprise manage their inventory independently, each enterprise has its own inventory. As can be seen from Figure 1, the JMI mode based on the supply chain is obviously superior to the traditional inventory management methods, the suppliers and manufacturers implement the raw materials joint inventory management and manufacturers and distributors implement the production and marketing joint stock. Upstream and downstream enterprises establish consultation management mechanism, the two sides work together to develop inventory plan, take the risk together, eliminating the phenomenon of all enterprises have their own stock, save the supply chain inventory costs.

\subsection{The Implementation Strategy of JMI.}

(1) Establish an effective coordination management mechanism

If JMI want to be able to give full play to the role of inventory management, upstream and downstream enterprises need to always cooperate with the business philosophy. When each node enterprises play their core competencies, it must establish a limited coordination management mechanism, it concludes: first, in addition to considering the enterprises themselves, each node enterprise should establish a common cooperation goal based on the principle of mutual benefit from the perspective of supply chain. Second, determine the optimal inventory method, that is, to determine the maximum inventory, the minimum inventory, demand forecasting, safety stock and more. Third, establish the mechanism of the distribution of benefits and incentive. To ensure the efficiency of cooperation and long-term cooperation among enterprises, a fair distribution of benefits and incentive mechanisms are essential. In addition, corresponding supervisory mechanisms need to be established to avoid the loss of profits caused by self-interest to other enterprises so as to enhance the efficiency of cooperation among enterprises.

(2) Establish information exchange system

Information exchange system refers to build a smooth channel for information transmission, and only guarantee the timely sharing of information in order to eliminate the adverse effects caused by distortion of demand distortion. The establishment of this information exchange system needs information technology, mainly including: EDI, ID barcode, continuous replenishment program, QR and so on. In addition, two kinds of resource planning systems should be put into effect, and the two most mature systems currently used in supply chain management are MPR II and DRP. By establishing an effective information system, the market demand information can be delivered in real time in the supply chain.

(3) Select the correct JMI mode

At present, the joint inventory management mode is mainly divided into two types: supplier keeps the inventory and the buyer keeps the inventory. Supplier keeps the inventory means that the demand-side reserves the ownership of a certain quantity of goods for a certain period of time by paying a deposit to the supplier, but the goods are still stored at the supplier's. When the buyer needs, the supplier provides the goods and the supplier shall bear the quantity, quality and other risks incurred before and during the delivery time. Buyer keeps the inventory refers to the supplier store the goods in the demander's warehouse according to the contract, both parties and suppliers are 
responsible for the daily management of the goods. The supplier assumes the responsibility of quality. The natural damage in the inventory shall be agreed upon by both parties.

(4) Make full use of third-party logistics

JMI can also rely on third-party logistics, that is, the logistics services will be provided by a professional logistics company. Third-party logistics companies can provide customers with a variety of professional logistics services, such as transportation, inventory management, so that enterprises can be more focused on the management of the core business.

\section{JMI Cost Model}

In figure 1, we can see that the suppliers and manufacturers can implement the raw materials joint inventory management and manufacturers and distributors can implement the production and marketing joint stock in JMI. Taking the production and marketing joint model as an example, we assume a two-level supply chain consisting of a manufacturer and a distributor and analyze the joint inventory costs.

\subsection{Model Assumptions.}

(1) The demand of distributors is continuous and uniform and the demand rate is constant;

(2) Both manufacturers and distributors use the EOQ order and are not allowed to stock out;

(3) Distributors adopt (0, Q) ordering strategy, regardless of order lead time.

\subsection{The Variables and Parameters of JMI Model.}

$\mathrm{K} 1$ : distributor unit product storage costs;

K2: manufacturer unit product storage costs;

K3: joint storage unit product storage costs;

$\mathrm{C} 1$ : distributor ordering costs;

C2: Manufacturer order processing Costs;

D: distributor's annual demand;

Q: distributor order quantity;

\subsection{The Establishment of Cost Model.}

\subsubsection{Traditional Supply Chain Inventory Costs.}

From the above assumptions, we can see that the distributor adopts $(0, Q)$ inventory strategy, so it does not need to consider the safety stock and lead time and does not consider the out-of-stock cost, so ordering cost $=\frac{D}{Q} C$, storage cost $=\frac{K Q}{2}$, total cost $=$ ordering cost + storage cost $=\frac{D}{Q} C+\frac{K Q}{2}$.

From the above, the total cost per unit time of the distributor in the traditional supply chain TC1= the ordering cost of the distributor + storage cost $=\frac{D C_{1}}{Q}+\frac{K_{1} Q}{2}$, find the first derivative of $\mathrm{Q}$ and TC (Q) $=0$, can get

$$
Q^{*}=\sqrt{\frac{2 D C_{1}}{K_{1}}}
$$

Substituting $\mathrm{Q}^{*}$ into the formula $\frac{D C_{1}}{Q}+\frac{K_{1} Q}{2}$, we can get $T C$ min $=\sqrt{2 D K_{1} C_{1}}$.

Manufacturers passively accept orders from distributors, so

$$
\begin{aligned}
& T C_{2}=\frac{D C_{2}}{Q^{*}}+\frac{Q^{*} K_{2}}{2}=\sqrt{2 D C_{1} K_{1}}\left(\frac{C_{2}}{2}+\frac{K_{2}}{2 K_{1}}\right) \\
& T C_{\text {tradition }}=\sqrt{2 D K_{1} C_{1}}+\sqrt{2 D C_{1} K_{1}}\left(\frac{C_{2}}{2}+\frac{K_{2}}{2 K_{1}}\right)
\end{aligned}
$$

\subsubsection{Joint Inventory Costs.}

After implementing joint inventory management, the total unit cost includes the distributor ordering costs, manufacturer order processing costs, and joint stock unit storage costs, so , 


$$
T C_{3}=\frac{D C_{1}}{Q}+\frac{D C_{2}}{Q}+\frac{K_{3} Q}{2}
$$

Find the first derivative of $\mathrm{Q}$ and TC3 $(\mathrm{Q})=0$, can get

$$
\mathrm{Q} 1 *=\sqrt{\frac{2 D\left(C_{1}+C_{2}\right)}{K_{3}}}
$$

Substitute $\mathrm{Q}^{*}$ into the formula, can get $\mathrm{TC}$ गмI $=\sqrt{2 D K_{3}\left(C_{1}+C_{2}\right)}$.Obviously, (TCлмI) ${ }^{2}<$ (TCTradition) ${ }^{2}$, this shows that all enterprises hold their own stocks, making the high cost of supply chain. This not only reduces the efficiency of the supply chain, but also increases the fund occupation. After the implementation of joint inventory management, supply chain inventory costs decline, the supply chain efficiency, inventory turnover rate also increased.

\section{Conclusion}

Joint inventory management can avoid distortion and enlargement of demand information. Through establishing a coordination mechanism and an effective information system, each node enterprise enhances the degree of enterprise information sharing and stock synchronization. The upstream and downstream enterprises in the supply chain unite as a whole maintain a high degree of strategic cooperative relations through close cooperation. At the same time, we can see from the cost model that JMI not only can reduce the inventory costs of the entire supply chain and optimize the supply chain system, but also can enhance the stability of the entire supply chain.

\section{References}

[1]. JIANG Hua, ZHANG Yun-chao, Yang Jing, The cost of JMI based on supply chain [J], and 2014(12):33-35.

[2]. Zhang Ganglia, Joint Inventory Management Appliance in the Supply Chain Inventory Control [J], 2008(4):14-15.

[3]. NI Zhi-wei, ZHU Xinhua, WU Zhang-jun, Inventory and distribution joint optimization model for muiti-cycle supply chain under stochastic demand[J], 2015(38):121-126.

[4]. Hong Dequan, Goo Wei, Study on Joint Managed Inventory Model Under No-shortage Based on Construction Materials Supply Chain[J], 2014(28):48-52.

[5]. Deng Hue, Research on Joint Inventory Management in Supply Chain Environment [J], 2016(31):44-47. 